\title{
Dynamic Origin of Vortex Core Switching in Soft Magnetic Nanodots
}

\author{
Konstantin Yu. Guslienko, Ki-Suk Lee, and Sang-Koog Kim* \\ Research Center for Spin Dynamics \& Spin-Wave Devices, Seoul National University, Seoul 151-744, Republic of Korea \\ Nanospintronics Laboratory, Department of Materials Science and Engineering, College of Engineering, Seoul National University, \\ Seoul 151-744, Republic of Korea
}

(Received 9 August 2007; revised manuscript received 1 November 2007; published 16 January 2008)

The magnetic vortex with in-plane curling magnetization and out-of-plane magnetization at the core is a unique ground state in nanoscale magnetic elements. This kind of magnetic vortex can be used, through its downward or upward core orientation, as a memory unit for information storage, and thus, controllable core switching deserves some special attention. Our analytical and micromagnetic calculations reveal that the origin of vortex core reversal is a gyrotropic field. This field is induced by vortex dynamic motion and is proportional to the velocity of the moving vortex. Our calculations elucidate the physical origin of the vortex core dynamic reversal, and, thereby, offer a key to effective manipulation of the vortex core orientation.

DOI: 10.1103/PhysRevLett.100.027203

PACS numbers: 75.40.Gb, 75.40.Mg, 75.60.Jk, 75.75.+a

Vortex patterns exist in physical dynamic systems of greatly varying temporal and spatial scales, ranging from quantized vortices in superfluids and superconductors [1] to water whirlpools, atmospheric tornadoes, and galaxies of the universe. These patterns reveal generic similarities such as the circular (spiral) structure of some parameters (density, velocity, etc.) and a vortex core. Intervortex forces and vortex inertial force (mass) are also somewhat similar in different vortex systems, because the vortices can often be treated as particles (solitons). The governing equations usually are nonlinear. Magnetic vortices are among the most prominent examples of the magnetization (M) ground states typically observed in submicron magnetic particles, such as nanodots [2-4]. Control of the $\mathbf{M}$ reversal in small particles is on the cutting edge of modern nanomagnetism. Vortices are elementary objects that describe the $\mathbf{M}$ reversal in such particles via their nucleation, propagation, and annihilation [5]. The magnetic vortex consists of the core (VC), a small area of $10-20 \mathrm{~nm}$ radius $R_{c}$ wherein $\mathbf{M}$ deviates from the dot plane [2,3], and the main part with the in-plane curling $\mathbf{M}$ around its core area. All known vortices are topological solitons or topological "defects" described by circulation, winding number, vorticity, etc. Magnetic vortices have topological charges of two different types [6], vorticity $q$ and polarization $p$, whereas other vortices usually have only one type. Existence of the VC nonzero topological charges $[1,6]$ in combination with the long-range magnetostatic interaction leads to unique effects in vortex dynamics. Nontrivial vortex excitations emerge that exist neither in bulk magnetic systems nor in continuous films. The vortex in nanodots possesses, in particular, a dynamic excitation, corresponding to the rotation of its core around an equilibrium position at a characteristic frequency of several hundred $\mathrm{MHz}$ [7-13]. The gyroforce responsible for this excitation, being perpendicular to the vortex velocity, is formally similar to the Magnus force acting on vortices in hydro- (aero-) dynamics, superfluids, and superconductors, but is of different physical origin. The unique nature of the vortex $\mathbf{M}$ distribution is expected to enable applications in magnetic data storage [14-18], spin wave generation [19], and other areas. For instance, a magnetic vortex can be used as an information carrier, because it has two discrete states of core orientation (up and down) and two directions of in-plane $\mathbf{M}$ rotation.

Very recently, the $\mathrm{VC}$ switching in magnetic nanodots (see Fig. 1) was experimentally and numerically demonstrated using a small-amplitude field pulse [14,20-22], as well as a spin-polarized current $[15,16]$. It was supposed that the switching occurs through vortex-antivortex pair creation and annihilation [14,16,20-22]. However, despite the considerable interest in dynamic VC switching, the origin of this new phenomenon has remained undiscovered. However, in this Letter, we are able to explain the physical origin of the $\mathrm{VC}$ orientation reversal. That is,

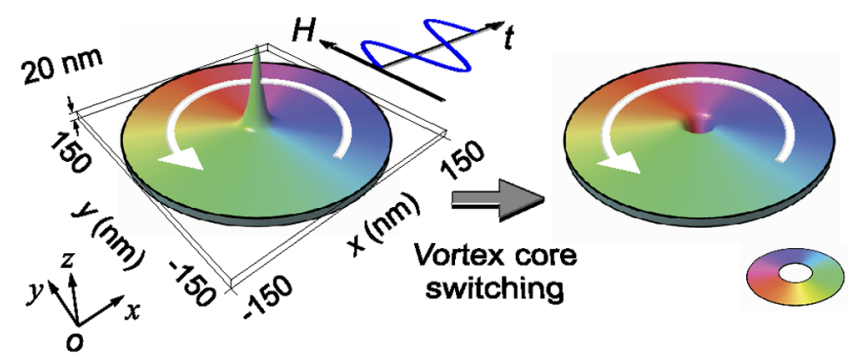

FIG. 1 (color online). Geometry of the model and scheme of the $\mathrm{VC}$ reversal in a cylindrical magnetic nanodot. The in-plane $\mathbf{M}$ distribution is marked by different colors, as indicated by the color wheel. The spikes correspond to the core magnetization: the switching occurs from the "upward" (left) to the "downward" orientation (right). The oscillating in-plane magnetic field amplitude is $H_{0}=50 \mathrm{Oe}$, and the field frequency is $\omega / 2 \pi=$ $580 \mathrm{MHz}$. 
whereas before we knew only how the reversal takes place, now we can also describe why it occurs.

In order to explain the physical origin of the $\mathrm{VC}$ orientation reversal, we consider the dynamic vortex $\mathbf{M}$ distribution $\mathbf{m}(\mathbf{r}, t)=\mathbf{M}(\mathbf{r}, t) / M_{s}$, where $M_{s}=|\mathbf{M}(\mathbf{r}, t)|$ is the saturation magnetization, and $\mathbf{r}$ is the coordinate in the dot plane. The $\mathbf{M}$ dependence on the coordinate along the nanodot thickness is neglected because of its small thickness, $\sim 10 \mathrm{~nm}$ (Fig. 1). We assume that the dot radius $R \gg R_{c}$ and that the vortex is the ground state. Shrinking $R$ up to $R_{c}$ leads to gradual destabilization of the vortex state and its transformation into a single-domain state [5].

We represent the time-derivative term in the magnetization equation of motion (the Landau-Lifshitz equation; see Ref. [23]) by a "gyrotropic" field $\mathbf{h}$. This is a novel and very useful way to describe VC switching. The gyrotropic field $\mathbf{h}$ and the corresponding torque can be calculated explicitly for a moving $\mathbf{M}$ vortex. The $\mathbf{h}$ is proportional to the vortex velocity $\mathbf{v}$ or to the shift of the VC position $\mathbf{X}(t)$ from an equilibrium position in a nanodot. The equation of the magnetization $\mathbf{M}$ motion can be formulated, in general, via an effective field, which is a variational derivative of the corresponding Lagrangian $L=$ $\int d^{3} \mathbf{x} \ell(\mathbf{m}, \dot{\mathbf{m}})$ with respect to $\mathbf{m}$ [23]. The Lagrangian density $\ell$ is equal to $g-w$, where $g$ is the kinetic (gyrotropic) term containing the time derivative of magnetization $\mathbf{m}$, and $w$ is the magnetic energy density. To define the kinetic part of the effective field, which we call the gyrotropic field or gyrofield, we use the kinetic part of the Lagrangian density in a form similar to that in the theory of spin coherent states [24]:

$$
g=\frac{M_{s}}{\gamma} \frac{\mathbf{n} \cdot(\mathbf{m} \times \dot{\mathbf{m}})}{1+\mathbf{m} \cdot \mathbf{n}},
$$

where $\gamma$ is the gyromagnetic ratio, and $\mathbf{n}$ is an arbitrary unit vector.

The Lagrangian density Eq. (1) has some singularity along the line $\mathbf{m} \cdot \mathbf{n}=-1$ [25], breaking rotation invariance. We assume that $\mathbf{n}$ describes the VC magnetization orientation, and we therefore choose $\mathbf{n}=\hat{\mathbf{z}}$ for the vortex with the core magnetization directed perpendicularly to the nanodot plane (Fig. 1). It is easy to confirm that in the representation of $\mathbf{m}$ via its spherical angles $\Theta, \Phi$, Eq. (1) reduces to the well-known form $g=\left(M_{s} / \gamma\right)(1-\cos \Theta) \dot{\boldsymbol{\Phi}}$ [23]. Let us define the VC polarization $p$ as the sign of the vortex $m_{z}$ component in its extreme point (the core magnetization orientation) $[1,6]$. We use Eq. (1) in the form $g=\left(M_{s} / \gamma\right)(\mathbf{m} \times \mathbf{m})_{z} /\left(p+m_{z}\right)$, which corresponds to the singularity [25] located along the negative or positive $O z$ semiaxis for $p=+1 /-1$ and define the gyrofield as $\mathbf{h}=M_{s}^{-1} \partial g / \partial \mathbf{m}$. Then, its $z$ component interacting with the $\mathrm{VC}$ can be expressed as

$$
h_{z}=-\frac{1}{\gamma} \frac{(\mathbf{m} \times \dot{\mathbf{m}})_{z}}{\left(m_{z}+p\right)^{2}} .
$$

Equation (2) in the angular representation is reduced to $h_{z}=-[\tan (\Theta / 2)]^{2 p} \dot{\boldsymbol{\Phi}} / \gamma$, and the singularity [25] is located along the line $\Theta=\pi$ or $\Theta=0$ for $p= \pm 1$, respectively. The gyrofield $\mathbf{h}$ is time dependent and, in particular, for a moving vortex $\mathbf{m}(\mathbf{r}, t)=\mathbf{m}(\mathbf{r}-\mathbf{X}(t))$, is proportional to the vortex velocity $\mathbf{v}=\dot{\mathbf{X}}$ introduced by the equation $\dot{\mathbf{m}}=-(\mathbf{v} \cdot \nabla) \mathbf{m}$. We consider that its $z$ component is the origin of the vortex profile $m_{z}(\mathbf{r}, t)$ dynamical deformation [12] (see Fig. 2 and supplementary movies 1 and 2 [27]). That deformation, leading eventually to the $\mathrm{VC}$ reversal and the $\mathrm{VC}$ velocity drop (shown in Fig. 3), is calculated numerically in Refs. $[15,16]$. The calculated $\mathbf{h}$ and torque [19] are concentrated near the VC, where the vortex magnetization component $m_{z}$ essentially differs from zero. That is, the dynamic core profile deformation up to the VC reversal is caused by the "effective" Zeeman energy, $w_{Z}(t)=-M_{s} h_{z}(t) m_{z}(t)$, which is indeed the driving force of the process. The $\mathbf{h}$ deforms the VC magnetization distribution $m_{z}(\mathbf{r}, t)$, creating a dip on the inner side of the core to match the field profile $h_{z}(\mathbf{r}, t)$ (Fig. 2). Assuming a circular VC motion of angular frequency $\boldsymbol{\omega}=$ $\omega \hat{\mathbf{z}}$ driven by an external in-plane oscillating field (Figs. 1 and 3) in a circular nanodot, the equation $\mathbf{v}=\boldsymbol{\omega} \times \mathbf{X}$ holds [28] and the $z$ component of the gyrofield is $h_{z}(\mathbf{r}, t)=(\omega / \gamma) F(r)[\hat{\mathbf{r}} \cdot \mathbf{X}(t)] / R_{c}$ (here $\hat{\mathbf{r}}$ indicates the unit vector along $\mathbf{r}$ ) within the linear approximation on the small ratio $|\mathbf{X}| / R$. The spatial distribution of the field reveals a different $h_{z}$ sign depending on the core coordinates. The gyrotropic field changes sign on the line perpendicular to $\mathbf{X}$, as shown in Fig. 2. The radial function $F(r)$ depends on the detailed VC profile and reaches its maximum $F(r) \approx 1$ within the $\mathrm{VC}, r<R_{c}$. This allows for the estimation of the maximum value of the gyrofield as $h_{z} \approx(\omega / \gamma)|\mathbf{X}| / R_{c}$. This value is about $1 \mathrm{kOe}$ near the VC and is sufficient to create an essential deformation of the VC magnetization profile, whereas $h_{z}$ outside the core is only $\sim 10$ Oe. Such a large and spatially nonuniform gyrofield interacting with the VC leads to its significant deformation and, eventually, to the core orientation switching at some critical value $h_{c} \sim v_{c}$ (where $v_{c}$ is the critical vortex velocity; see Fig. 3). The vortex velocity $v$ is proportional to the oscillating field or spin-polarized current amplitude, whereas the critical velocity $v_{c}$ is an intrinsic parameter for the given nanodot geometry and does not depend on the driving force parameters [16]. We estimate $v_{c}$ as $v_{c} \approx$ $\gamma M_{s} R_{c} \approx \gamma(2 A)^{1 / 2}$ ( $A$ is the exchange stiffness), which is about $320 \mathrm{~m} / \mathrm{s}$ for the Permalloy nanodot. This estimation of $v_{c}$ is in good agreement with numerical simulations [29], as shown in Fig. 3(b). In some sense, the $v_{c}$ is similar to the Walker critical velocity for domain walls [23], because it corresponds to a transition from the linear gyrotropic motion to the strongly nonlinear regime of the $\mathrm{VC}$ orientation oscillations. Under the influence of an applied oscillating driving field, the VC amplitude $|\mathbf{X}(t)|$ and velocity increase with time [see Figs. 3(b) and 3(c) and supplementary movie 3 [27]), and when they reach some 


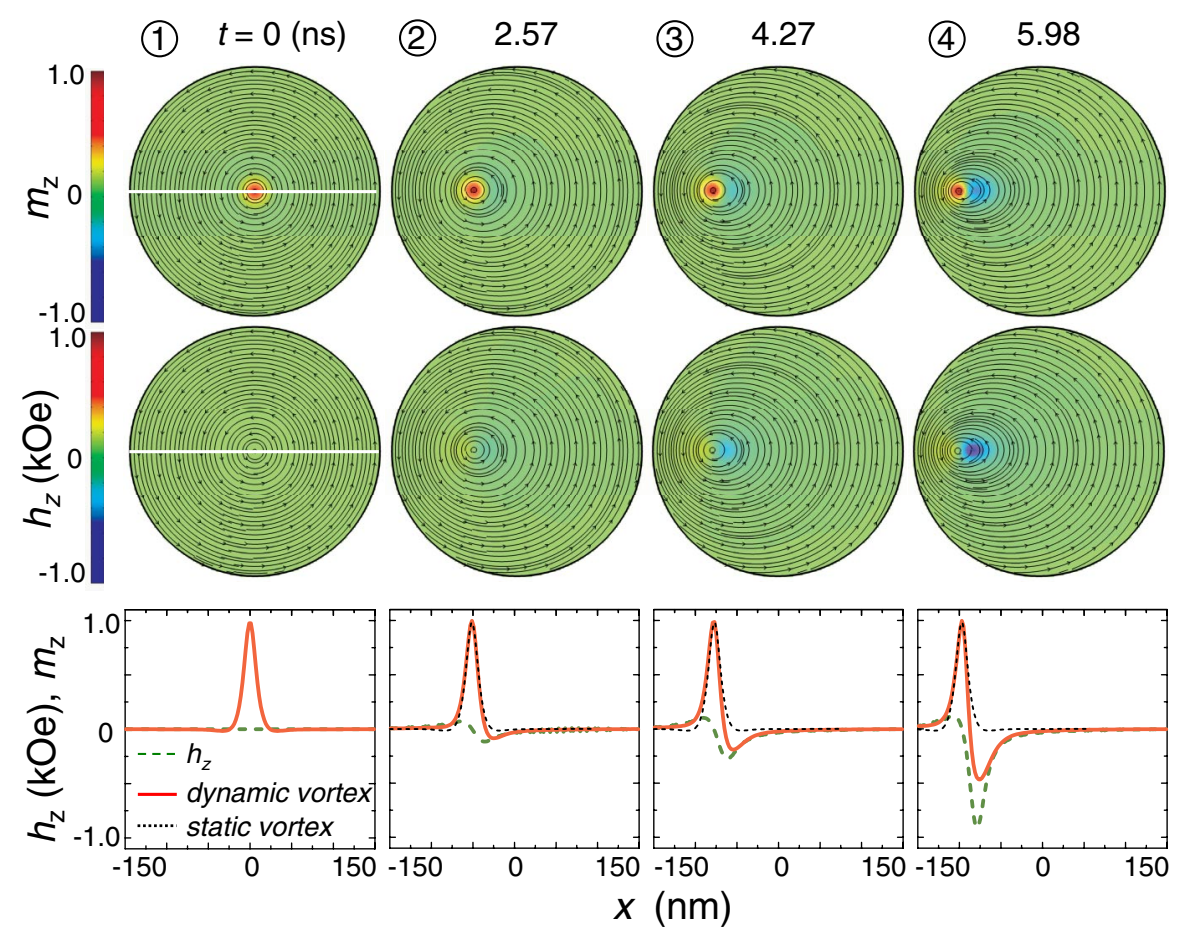

FIG. 2 (color online). Snapshot images at the selected moments of the spatial $\mathbf{M}$ distribution and of the gyrofield distribution for a moving vortex. The color of the surfaces indicates the local out-ofplane normalized magnetization $m_{z}$ in the first row and the local out-of-plane component $h_{z}$ in the second row. The streamlines with the small arrows indicate the in-plane $\mathbf{M}$ direction. The VC magnetization [solid (red) line] and the gyrofield $z$ component [dashed (green) line] profiles along the line crossing the $\mathrm{VC}$ and the nanodot center are shown in the third row. The static vortex profile [dotted (black) line] was intentionally shifted for direct comparison with the dynamic vortex profile [solid (red) line]. critical values, the VC suddenly reverses its polarization. That is, the moving vortex adsorbs the energy of the oscillating driving field and therefore increases its profile deformation and velocity. Then, the energy excess is emitted in the form of spin waves [19], and the vortex velocity and oscillation amplitude sharply decrease [Figs. 3(b) and 3 (c)]. In the case of a periodic driving field, this $\mathrm{VC}$ reversal process also repeats periodically. This effect has been numerically observed and used to generate strong spin waves immediately following the VC reversal [19,22].

To confirm the physical mechanism of the VC reversal as driven by an oscillating magnetic field, we conducted micromagnetic calculations $[29,30]$ and showed that the spatial distribution of $h_{z}$ (Fig. 2) is indeed close to the
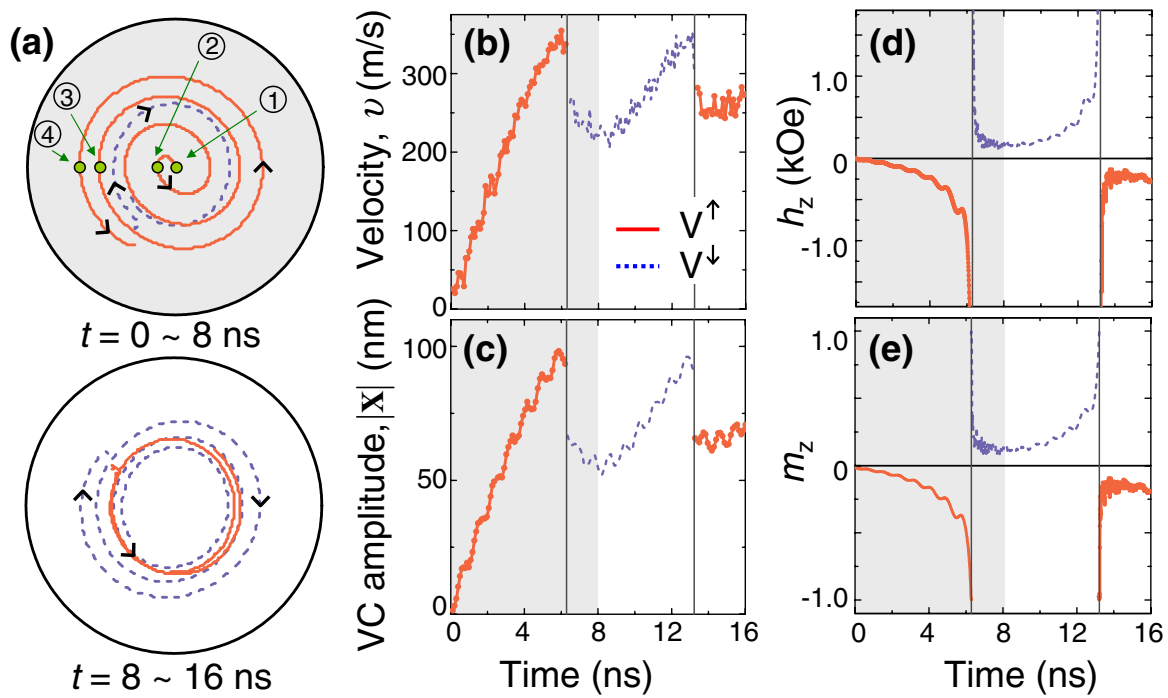

FIG. 3 (color online). (a) The simulated trajectories of the VC motion under an oscillating magnetic field during the time periods $t=0-8 \mathrm{~ns}$ for the upper panel (shaded circle) and $t=8-16 \mathrm{~ns}$ for the lower panel. The small circles (green) indicate the VC positions at the times indicated for the snapshot images in Fig. 2. The velocity $v$ and the amplitude $|\mathbf{X}|$ of the moving VC are plotted vs time in (b) and (c), respectively. VC orientation reversal occurs at the maximum velocity $\left(v_{c}\right)$ and amplitude values. The extreme minimum value of the local $h_{z}$ for the upward core orientation and its maximum value for the downward core orientation and the local $m_{z}$ are shown vs time in (d) and (e), respectively. The solid (red) and dashed (blue) lines correspond to the upward $(p=+1)$ and downward $(p=-1)$ core orientations, respectively. The vertical lines indicate the times when the extreme values of the local $h_{z}$ have infinite values, which correspond to the singularity of the gyrofield Eq. (2) at the points $m_{z}=-p$. 
distribution of $m_{z}(r, t)$. For comparison, the vortex dynamic $\mathbf{M}$ distribution is plotted in Fig. 2, which clearly illustrates that the $\mathrm{VC}$ dynamic distortion profile follows the distribution of the $z$ component of the gyrotropic field. If we consider the time evolution of the extreme value of the gyrofield [Fig. 3(d)] and the out-of-plane $\mathbf{M}$ component $m_{z}$ [Fig. 3(e)], it is also evident that the component $m_{z}(t)$ practically follows the dependence $h_{z}(t)$. The gyrofield $h_{z}$, in accordance with our Eq. (2), reaches infinite values in the time moments of the VC reversal [see Fig. 3(d)] due to our definition of this field by Eq. (2). Other fields, such as the exchange and magnetostatic fields, also contribute to the VC dynamics, but, namely, the gyrofield is crucial to the reversal, being its driving force. The consideration of the gyrofield above is valid for any static VC profile as well as for any ansatz of the $\mathbf{M}$ distribution of the moving vortex in the form $\mathbf{m}(\mathbf{r}, t)=\mathbf{m}(\mathbf{r}, \mathbf{X}(t))$. The distortion of the moving $\mathrm{VC}$ profile due to the gyrofield, and the resulting $\mathrm{VC}$ orientation reversal, are general phenomena for any dot shape. That dynamical distortion has been observed, in particular, in square dots [14] and circular dots [15] excited from the centered $\mathbf{M}$ vortex ground state.

The results here presented offer insight into VC reversal as driven by either a small-amplitude in-plane oscillating magnetic field or a spin-polarized current. Understanding of the physical origin of vortex core reversal is the key to the effective manipulation of the dynamic switching of core orientation via the choice of appropriate strengths and frequencies of driving forces as well as of magnetic nanoelement geometry.

This work was supported by Creative Research Initiatives (Research Center for Spin Dynamics \& SpinWave Devices) of MOST/KOSEF.

*Corresponding author. sangkoog@snu.ac.kr

[1] N. Manton and P. Sutcliffe, Topological Solitons (Cambridge University Press, Cambridge, 2004), Chap. 1.

[2] T. Shinjo et al., Science 289, 930 (2000).

[3] A. Wachowiak et al., Science 298, 577 (2002).

[4] R. P. Cowburn et al., Phys. Rev. Lett. 83, 1042 (1999).

[5] K. Yu. Guslienko et al., Phys. Rev. B 65, 024414 (2002).

[6] A. M. Kosevich, B. A. Ivanov, and A.S. Kovalev, Phys. Rep. 194, 117 (1990).

[7] K. Yu. Guslienko et al., J. Appl. Phys. 91, 8037 (2002).

[8] J. P. Park et al., Phys. Rev. B 67, 020403(R) (2003).

[9] S. B. Choe et al., Science 304, 420 (2004).

[10] X. Zhu et al., Phys. Rev. B 71, 180408(R) (2005).

[11] K. Yu. Guslienko et al., Phys. Rev. Lett. 96, 067205 (2006).

[12] V. Novosad et al., Phys. Rev. B 72, 024455 (2005).

[13] K.-S. Lee and S.-K. Kim, Appl. Phys. Lett. 91, 132511 (2007).
[14] B. Van Waeyenberge et al., Nature (London) 444, 461 (2006).

[15] K. Yamada et al., Nat. Mater. 6, 270 (2007).

[16] S.-K. Kim et al., Appl. Phys. Lett. 91, 082506 (2007).

[17] R. P. Cowburn, Nat. Mater. 6, 255 (2007).

[18] J. Thomas, Nature Nanotechnology 2, 206 (2007).

[19] S. Choi et al., Phys. Rev. Lett. 98, 087205 (2007); K.-S. Lee et al., Appl. Phys. Lett. 87, 192502 (2005).

[20] R. Hertel, S. Gliga, M. Fähnle, and C. M. Schneider, Phys. Rev. Lett. 98, 117201 (2007).

[21] Q.F. Xiao et al., Appl. Phys. Lett. 89, 262507 (2006).

[22] K.-S. Lee, K. Y. Guslienko, J.-Y. Lee, and S.-K. Kim, Phys. Rev. B 76, 174410 (2007).

[23] A. Hubert and R. Schafer, Magnetic Domains (SpringerVerlag, Berlin, 1998), Sec. 3.6.6.

[24] A. Auerbach, Interacting Electrons and Quantum Magnetism (Springer-Verlag, Berlin, 1994), Chap. 10.

[25] The singularity corresponds to Dirac's string along the direction $\mathbf{n}$, which is related to the magnetic monopole and is closely related to the Berry phase. If we use the variable-length magnetization $\mathbf{M}^{\prime}=M \mathbf{m}$ (without constraint $\left.\left|\mathbf{M}^{\prime}\right|=M_{s}\right)$, then we can write $g=\mathbf{A} \cdot \dot{\mathbf{M}}^{\prime}$, where the vector potential is $\mathbf{A}=\left(M_{s} / \gamma\right)\left[\mathbf{n} \times \mathbf{M}^{\prime}\right] / M(M+\mathbf{n}$. $\mathbf{M}^{\prime}$ ) [26]. This potential is singular along the line $\mathbf{M}^{\prime} \cdot \mathbf{n}=$ $-M$. The effective magnetic field $\mathbf{B}=\nabla_{\mathrm{M}^{\prime}} \times \mathbf{A}=$ $\left(M_{s} / \gamma\right) \mathbf{M}^{\prime} / M^{3}$ describes a magnetic monopole in $\mathbf{M}^{\prime}$ space with the "magnetic" charge $M_{s} / \gamma$ located at the origin $\mathbf{M}^{\prime}=\mathbf{0}$. We interpret a magnetic vortex as the crossing of the Dirac string with the surface of the sphere $\left|\mathbf{M}^{\prime}\right|=M_{s}$ in $\mathbf{M}^{\prime}$ space. This leads to the kinetic part of the Lagrangian density in the form of Eq. (1).

[26] F. D. M. Haldane, Phys. Rev. Lett. 57, 1488 (1986); B. A. Ivanov and N. E. Kulagin, J. Exp. Theor. Phys. 99, 1291 (2004).

[27] See EPAPS Document No. E-PRLTAO-100-054802 for the three supplementary movie files. For more information on EPAPS, see http://www.aip.org/pubservs/epaps.html.

[28] The typical VC dynamic shift in submicron size dots is $|\mathbf{X}| \sim 100 \mathrm{~nm}$, and the vortex velocity is about $100 \mathrm{~m} / \mathrm{s}$, which is comparable to the velocity of moving domain walls.

[29] We used the following material and geometrical parameters for the Permalloy nanodot: $M_{s}=860 \mathrm{G}$, the exchange stiffness $A=1.3 \mu \mathrm{erg} / \mathrm{cm}$, the damping constant $\alpha=0.01, \gamma=17.6 \mathrm{MHz} / \mathrm{Oe}$, the dot radius of $150 \mathrm{~nm}$, and thickness of $20 \mathrm{~nm}$ (Fig. 1). The vortex motion was driven by an external oscillating in-plane field $\mathbf{H}(t)=H_{0} \sin (\omega t) \hat{\mathbf{y}}$ with the amplitude $H_{0}=50$ Oe. In order to effectively excite the vortex gyrotropic motion, we chose $\omega / 2 \pi=580 \mathrm{MHz}$, which is close to the vortex eigenfrequency for the given dot material and geometry. The critical vortex velocity was numerically calculated to be $v_{c}=350 \pm 20 \mathrm{~m} / \mathrm{s}$ for the nanodot parameters.

[30] M. Donahue and D. Porter, OOMMF User's Guide, version 1.0. Interagency Report No. NISTIR 6376, National Institute of Standards and Technology, Gaithersburg, MD, 1999. 